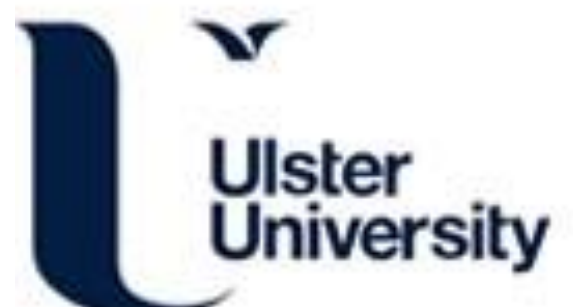

\section{Pulsed electromagnetic energy (PEME) in management of chronic wounds: a systematic review}

McGaughey, H., Dhamija, S., Oliver, L., Porter-Armstrong, A., \& McDonough, S. (2009). Pulsed electromagnetic energy (PEME) in management of chronic wounds: a systematic review. Physical Therapy Reviews, 14(2), 132146. https://doi.org/10.1179/174328809X435231

Link to publication record in Ulster University Research Portal

\section{Published in:}

Physical Therapy Reviews

Publication Status:

Published (in print/issue): 01/04/2009

\section{DOI:}

10.1179/174328809X435231

\section{Document Version}

Publisher's PDF, also known as Version of record

\section{General rights}

Copyright for the publications made accessible via Ulster University's Research Portal is retained by the author(s) and / or other copyright owners and it is a condition of accessing these publications that users recognise and abide by the legal requirements associated with these rights.

\section{Take down policy}

The Research Portal is Ulster University's institutional repository that provides access to Ulster's research outputs. Every effort has been made to ensure that content in the Research Portal does not infringe any person's rights, or applicable UK laws. If you discover content in the Research Portal that you believe breaches copyright or violates any law, please contact pure-support@ulster.ac.uk. 


\section{Pulsed electromagnetic energy in management of chronic wounds: a systematic review}

\section{Helen McGaughey ${ }^{1}$, Sue Dhamija ${ }^{1}$, Louise Oliver $^{2}$, Alison Porter-Armstrong ${ }^{1}$ and Suzanne McDonough ${ }^{1}$}

${ }^{1}$ Health and Rehabilitation Sciences Research Institute, School of Health Sciences, University of Ulster, Northern Ireland

${ }^{2}$ School of Psychology, Queen's University Belfast, Northern Ireland

Objectives: To assess the evidence regarding effectiveness of pulsed electromagnetic energy (PEME) on the healing of chronic wounds; to explore whether there is an optimum treatment regime with regards to total current, pulse amplitude, pulse duration, and duration and frequency of treatments.

Methods: A computerised literature search of the Cochrane Library, the Cumulative Index to Nursing and Allied Health Literature (CINAHL), EMBASE and PubMed was performed. Only randomised clinical trials (RCT), controlled trials, and clinical trials that studied a population aged 18 years of age or more and investigated the effects of a type of PEME in the management of a chronic wound were included. The methodological quality of the included trials was assessed qualitatively using a set of formal criteria as recommended by van Tulder et al.

Results: Eleven studies ( $n=360$ ) out of 40 were included of which six were of high methodological quality. Methodological scores ranged from one to nine (maximum 11) with a mean score of 5.5 $(S D=1 \cdot 73)$. For venous ulcers, there is strong evidence that PEME is more effective than sham PEME. For pressure and plantar ulcers, there is moderate evidence that PEME, in combination with conventional wound care is better than conventional wound care alone. The heterogeneity in duration, frequency, voltage and magnetic field made it difficult to make detailed comparisons or specific recommendations regarding its application.

Discussion: Further research should focus on controlling baseline recruitment parameters within an RCT, studying vital outcomes, and exploring combinations of parameters regarding optimum usage of PEME.

Keywords: Pulsed electromagnetic energy, chronic wounds, ulcer, systematic review

\section{Background}

Chronic wounds are defined as wounds that fail to heal in the expected time with conventional treatment; ${ }^{1}$ the three main types are lower limb ulcers of vascular aetiology (arterial, venous and mixed ulcers), diabetic ulcers and pressure ulcers. Skin ulcers are a complex group of disorders which take a prolonged period of time to heal, do not heal, or reoccur frequently. The prevalence and economic cost of wound treatment is continually increasing and as a result the patient, their families and the health professionals involved, face significant medical, social and economic problems. ${ }^{2}$

An ulcer is a breach in the continuity of an epithelium. Pressure ulcers (also known as pressure sores, bed ulcers, bed sores and decubitus ulcers) are 
defined as 'an area of localised damage to the skin and underlying tissue caused by pressure, shear, friction and/or a combination of these'. ${ }^{3}$ The estimated annual cost of treating pressure ulcers ranges from $£ 1 \cdot 4$ to $£ 2 \cdot 1$ billion, approximately $4 \%$ of the UK's total health care expenditure. ${ }^{4}$ The National Health Service Centre for Reviews and Dissemination ${ }^{5}$ states that the estimated prevalence of leg ulcers in the UK is $1 \cdot 5-3$ per 1000 of the population, increasing to 20 per 1000 in people over 80 years of age. At a cost of approximately $£ 400$ million a year, ${ }^{6}$ leg ulcers constitute a considerable cost to both the patient ${ }^{7}$ and the health service. ${ }^{8}$ Venous ulcers account for about $70 \%$ of chronic ulcers and result from venous insufficiency. ${ }^{9}$ In comparison arterial ulcers of the lower limb constitute only 10 to $20 \%$ of leg ulcers ${ }^{10}$ and are caused by insufficient arterial blood supply resulting in tissue ischaemia and necrosis. ${ }^{11}$

There are several factors that can impede the three phases of wound healing (inflammation, proliferation and remodeling). ${ }^{12}$ They include malnutrition, infection, tissue hypoxia, age, ${ }^{13}$ metabolic derangements and drugs. ${ }^{14}$ Divided into three categories, the main groups of patients likely to suffer from chronic wounds are: the elderly, those with spinal cord injuries and people with peripheral vascular disease. $^{15}$

Research into the role of electricity in wound healing can be traced back as far as the $1940 \mathrm{~s} .{ }^{16}$ The medical use of high frequency electrical currents can be traced back as far as the $1890 \mathrm{~s}^{17}$ with pulsed electromagnetic energy (PEME) first introduced in the early 1950 s. $^{2}$ Hailed as a cure for many ailments it became popular very quickly with clinical therapists. ${ }^{2}$ It was initially introduced into the UK following the Mexico Olympic Games in 1968 during which a British physiotherapist observed the positive results of using diapulse on an injured athlete. ${ }^{18}$

Pulsed electromagnetic energy encompasses the terms pulsed short wave diathermy, pulsed electromagnetic field (PEMF) and diapulse. Short wave diathermy uses the radio frequency wave bands of $27 \cdot 12 \mathrm{MHz}$, one of three high frequency bands allocated for medical use that prevents interference with other bands used in communications. ${ }^{2}$ Pulsing creates side bands with energy frequencies ranging from 26.95 to $27.28 \mathrm{MHz}$ with little in the parent band of $27 \cdot 12 \mathrm{MHz}$. These pulse lengths vary from 25 to $400 \mu$ s and the number of pulses varies between 15 and $800 \mathrm{~s}^{-1} \cdot{ }^{19}$ In PEME the energy is delivered in a sequence of pulses with the 'off' period being much longer than the 'on' period ${ }^{20}$ resulting in a lower overall dose being given to the patient.
Electromagnetic therapy is a field effect and not a direct electrical effect or a form of radiation, thus making it distinct from most other forms of electrotherapy. Electromagnetic energy is made up of two components: the electric field (E-field) and the magnetic field (H-field). ${ }^{21}$ It has been proposed that cell oscillation caused by the E-field and alterations in cell potential and ionic interchange by the $\mathrm{H}$-field ${ }^{18}$ can account for some of the physiological effects of PEME.

Essentially, the aim of PEME is 'to give the tissues an energy boost in the form of an electromagnetic field without the tissues being required to tolerate a thermal load'. ${ }^{2}$ There are several theories underpinning the therapeutic usage of PEME, the simplest being that the electromagnetic energy 'stirs' ions, molecules, membranes and cells thus enhancing phagocytic and enzyme activity and transport across membranes. ${ }^{22}$ It is also proposed that the electromagnetic field can alter cell membrane potential, influencing the flow of ions across it, therefore restoring normal cell potential in damaged cells. ${ }^{21}$ The physiological effects are thought to include: an increase in the number of white cells and fibroblasts in a wound, improved rate of oedema dispersion and re-absorption of haematomas, reduction of inflammation, enhanced deposition and organisation of collagen and fibrin, stimulation of osteogenesis $^{21,23}$ and enhanced blood flow. ${ }^{24}$ Pulsed electromagnetic field may have the ability to influence a number of the processes involved in the healing of wounds but the extent to which wound healing rates are actually increased is unclear.

A survey of physiotherapists' use of electrophysical agents in Australia found that less than half of those who responded had shortwave diathermy available, very few used it daily and those who did tended to use continuous shortwave. ${ }^{25}$ In contrast, in England PEME is one of the most commonly used electrotherapy modalities within physiotherapy, ${ }^{26}$ despite the lack of standardisation regarding the type, duration, frequency and intensity of PEME implemented and ongoing questions regarding its clinical effectiveness. A number of studies have evaluated the effects of PEME on wound healing, but at this time no systematic review evaluating all chronic wounds has been performed. For such a well-utilised treatment, ${ }^{26}$ it is essential that the methodological quality of relevant literature be identified to enable evidencebased recommendations to be made.

\section{Objectives}

This review aims to systematically assess the evidence for any effect of pulsed electromagnetic energy on the 
healing of chronic wounds, and to explore how varying different parameters of stimulation, such as total current, pulse amplitude, pulse duration, treatment schedule etc affect healing. This review will seek to answer the following questions:

(i) short-term aims:

- Does pulsed electromagnetic energy therapy accelerate the rate of wound healing?

- If yes, what is the optimum treatment regimen with regards to total current, pulse amplitude, pulse duration, and duration and frequency of treatments?

(ii) long-term aim:

- To provide recommendations for clinical practice and further research.

\section{Methods}

\section{Search strategy for identification of studies}

Relevant studies were identified using a computerbased literature search of four databases. The Cochrane Database of Systematic Reviews, the Cochrane Wounds Group Specialised Register, the Cochrane Central Register of Controlled Trials and the Cochrane Database of Abstracts of Reviews of Effect (Cochrane) (last searched December 2008); PubMed Central (last searched December 2008); EMBASE on OVID (1980 to December 2008); and the Cumulative Index to Nursing and Allied Health Literature (CINAHL on OVID) (1982 to December 2008). There was no restriction on the basis of language, date of trial or publication status. The databases were searched using the highly sensitive search strategy recommended in the Cochrane Collaboration Handbook. ${ }^{27}$ The keywords used were: wound healing, pressure ulcer, pressure sore, decubitus ulcer, leg ulcer, varicose ulcer, diabetic foot, chronic ulcer, venous ulcer, electromagnetics, electromagnetic field, electric stimulation, diathermy, diapulse, magnetic therapy, magnetic field, pulsed stimulation, pulsed therapy, randomised controlled trial, clinical trial, case study and controlled trial. Keywords were combined together.

\section{Criteria used in considering studies for this review}

Types of studies: Randomised controlled trials (RCTs), controlled trials or clinical trials.

Types of participants: Studies involving human subjects aged 18 or over diagnosed with a chronic ulcer (pressure, venous leg, varicose, diabetic foot, arterial leg, mixed arterial, chronic, decubitus) or sore (pressure, bed).

Types of intervention: Any form of pulsed electromagnetic therapy for healing any type of wound compared with sham or a different intensity of pulsed electromagnetic therapy, no pulsed electromagnetic therapy or other treatments.

\section{Types of outcome measures:}

Primary outcomes: wound healing defined by objective measures such as:

- time to complete healing

- proportion of wound healed within the trial period

- rate of change in wound size (surface area, depth or volume).

\section{Methods of review}

In the first stage of selection the titles and abstracts of identified articles were assessed according to the eligibility criteria. A study was excluded if it was clear from the title and/or abstract that it was not relevant. The full article was obtained if the study's degree of relevance was unclear or the abstract for a title deemed relevant was not available. Full papers were checked to identify those eligible for inclusion. Another author repeated this process to provide verification. Any disagreements were resolved by discussion and if necessary referred to a third reviewer for adjudication. The reference lists of selected articles were reviewed for additional relevant studies.

\section{Methodological quality}

The methodological quality of each study and quality of reporting was assessed using a set of formal criteria (Table 1) and applied using a uniform operationalisation of criteria (Table 2) as recommended by van Tulder et $a l .{ }^{28}$ Only the criteria pertaining to internal validity were applied. A pilot test of methodological quality assessment, recommended by van Tulder et al. ${ }^{29}$ was performed on selected articles not included in the review to ensure both reviewers agreed on a common interpretation of the scale items and their operationalisation. The van Tulder scale was chosen as it includes all the criteria of Jadad et al. ${ }^{30}$ and Verhagen ${ }^{31}$ and measures all but one of the PEDRO scale criteria. ${ }^{32}$ Each criterion is scored yes $(\mathrm{Y})$, no $(\mathrm{N})$ or do not know (DK) with only an answer of yes scoring one point each. A study was considered high quality if it fulfilled six or more of the 11 internal validity criteria. ${ }^{33}$ The studies were not blinded for authors, institutions or journals in which the studies were published. 
Table 1 Methodological quality ${ }^{28}$

\begin{tabular}{lll}
\hline A & Was the method of randomisation adequate? & Y/N/DK \\
$\mathrm{B}$ & Was the treatment allocation concealed? & Y/N/DK \\
$\mathrm{C}$ & Were the groups similar at baseline regarding most prognostic indicators? & Y/N/DK \\
$\mathrm{D}$ & Was the patient blinded to the intervention? & Y/N/DK \\
$\mathrm{E}$ & Was the care provider blinded to the intervention? & Y/N/DK \\
$\mathrm{F}$ & Was the outcome assessor blinded to the intervention? & Y/N/DK \\
$\mathrm{G}$ & Were co interventions avoided or comparable? & Y/N/DK \\
$\mathrm{H}$ & Was the compliance acceptable in all groups? & Y/N/DK \\
$\mathrm{I}$ & Was the dropout rate described and acceptable? & Y/N/DK \\
$\mathrm{J}$ & Was the timing of the outcome assessment in all groups similar? & Y/N/DK \\
$\mathrm{K}$ & Did the analysis include an intention-to-treat analysis? & Y/N/DK \\
\hline
\end{tabular}

\section{Data extraction and analysis}

The procedure for data collection and extraction was conducted in accordance with the guidelines described by van Tulder et al. ${ }^{28}$ Data was independently extracted and summarised from each study. The type of chronic wound, study population, study design, inclusion/exclusion criteria, outcome measures, co-interventions and study results were recorded. For each intervention, the mode, frequency, pulse rate, pulse duration, peak power, and duration of PEME applied were also recorded.

A qualitative analysis ('best evidence synthesis') was conducted using a rating system consisting of four levels of evidence:

- Level 1- strong evidence: generally consistent findings in multiple high quality trials

- Level 2 - moderate evidence: generally consistent findings in multiple low quality trials and/or one high quality trial

- Level 3a - limited evidence: only one low quality trial

- Level 3b - conflicting evidence: inconsistent findings in multiple trials
- Level 4 - no evidence: no RCTs and no doubleblind trials.

Subgroup analyses were planned for the following:

(a) pressure ulcers

(b) venous ulcers

(c) plantar ulcers.

\section{Results}

On initial examination of the titles and abstracts yielded from the database search, 40 studies were included. Following review of the full text, 29 studies were excluded, resulting in 11 studies eligible for inclusion in the review. Figure 1 summarises the process of study selection and the number of studies excluded at each stage. Results are recorded in the quality of reporting of meta-analyses (QUORUM) statement flow diagram (Fig. 1). ${ }^{34}$

\section{Methodological quality}

The methodological quality of the studies (see Table 3) ranged from one point to nine points with a mean of $5 \cdot 5$ (standard deviation $1 \cdot 73$; median 6 ) out

Table 2 Operationalisation of the criteria ${ }^{28}$

A A random (unpredictable) assignment sequence. Examples of adequate methods are computer generated random number table and use of sealed opaque envelopes. Methods of allocation using date of birth, date of admission, hospital numbers, or alternation should not be regarded as appropriate.

B Assignment generated by an independent person not responsible for determining the eligibility of the patients. This person has no information about the persons included in the trial and has no influence on the assignment sequence or on the decision about eligibility of the patient.

C In order to receive a 'yes' groups must be similar at baseline regarding demographic factors, duration and severity of complaints, percentage of patients with neurologic symptoms, and value of main outcome measure(s).

D The reviewer determines when enough information about the blinding is given in order to score a 'yes'. The reviewer determines when enough information about the blinding is given in order to score a 'yes' The reviewer determines when enough information about the blinding is given in order to score a 'yes'. Co-interventions should either be avoided in the trial design or comparable between the index and control group. The reviewer determines if the compliance to the intervention is acceptable, based on the reported intensity, duration, number and frequency of sessions for both the index intervention and the control interventions.

I The number of participants included in the study but who did not complete the observation period or were not included in the analysis must be described and reasons given. If the percentage of withdrawals and dropouts does not exceed $20 \%$ for short-term follow-up and $30 \%$ for long-term follow-up and does not lead to substantial bias, a 'yes' is scored.

J Timing of outcome assessment should be identical for all intervention groups and for all important outcome assessments.

K All randomised patients are reported/analysed for the most important moments of effect measurement (minus missing values) irrespective of non-compliance and co-interventions 
No of Hits:

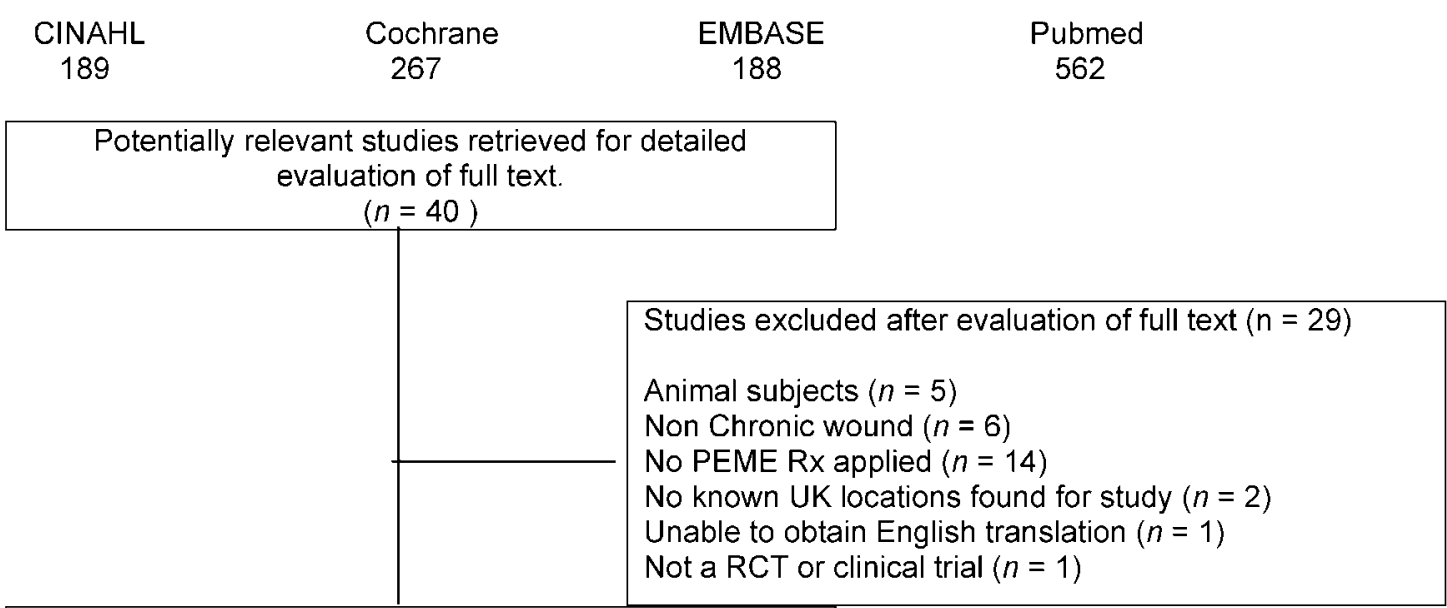

Studies eligible for inclusion in systematic review.

$$
(n=11)
$$

Figure 1 The quality of reporting of meta-analysis (QUORUM) statement flow diagram ${ }^{34}$

of 11. Overall, studies performed best in having comparable timing of outcome assessments ${ }^{35-43}$ avoiding the use of co-interventions ${ }^{35-37,39-45}$ and in adequately describing an acceptable dropout rate. $^{35,37-40,42-44}$ Blinding of the patient ${ }^{35,37-40,42-44}$ and outcome assessor ${ }^{35,37,39-43}$ to the intervention were also performed well. In contrast however, only $50 \%$ of studies blinded the care provider ${ }^{38,39,42-44}$ and just one used concealed allocation during recruitment. ${ }^{35}$ Although performed by eight studies $^{35,37-41,42,44}$ only three employed a satisfactory method of randomisation. ${ }^{35,41,42}$ In addition, only two studies adequately performed an intention-totreat analysis ${ }^{36,38}$ and despite a number of betweengroup comparisons being made throughout the studies, there was a high degree of group variability at baseline. ${ }^{35-38,40,41,43,44}$ It should be noted that failure to report on key information resulted in $27 \cdot 3 \%$ of the total internal validity criteria scoring a 'don't know'. Overall six studies $35,37,39,40,42,43$ in this review were of high quality scoring at least six points in internal validity criteria and five $\mathrm{e}^{36,38,41,44,45}$ were of low quality. There was no clear trend relating to publication date and time of publication.

\section{Study characteristics}

The study characteristics and the protocols employed within each study are shown in Tables 4 and 5 respectively. Eleven studies were included, using a total of 360 participants. Overall there were 183 male participants compared to 170 female participants, with two studies involving males only. ${ }^{39,41}$ The sex of seven of the participants is unknown, as one study ${ }^{40}$ failed to record the participants that withdrew or dropped out of the study. The mean number of subjects used was 32 , with the sample size ranging from 19 to 86 and the age of participants ranging from 20 to 101 years of age. In total just $8 \cdot 4 \%$ of all participants $(n=23)$ withdrew or dropped out of a study. Reasons cited included: not adhering to program parameters $(n=11)$, personal reasons $(n=3)$, ulcers revealed to be malignant $(n=3)$,

Table 3 Methodological quality scores

\begin{tabular}{|c|c|c|c|c|c|c|c|c|c|c|c|c|}
\hline Study & A & B & $\mathrm{C}$ & D & $E$ & $F$ & G & $\mathrm{H}$ & I & $J$ & $\mathrm{~K}$ & Total score \\
\hline Comorosan et al. ${ }^{44}$ & DK & DK & $\mathrm{N}$ & Y & Y & DK & Y & DK & Y & $\mathrm{N}$ & $\mathrm{N}$ & 4 \\
\hline leran et al. ${ }^{35}$ & Y & $\mathrm{Y}$ & $\mathrm{N}$ & $Y$ & Y & $\mathrm{Y}$ & Y & Y & Y & $Y$ & $\mathrm{~N}$ & 9 \\
\hline Itoh et al. ${ }^{36}$ & $\mathrm{~N}$ & DK & $\mathrm{N}$ & $\mathrm{N}$ & $\mathrm{N}$ & DK & Y & DK & Y & Y & Y & 4 \\
\hline Jeran et al. ${ }^{37}$ & DK & DK & $\mathrm{N}$ & Y & DK & $\mathrm{Y}$ & Y & Y & Y & Y & $\mathrm{N}$ & 6 \\
\hline Kenkre et al. ${ }^{38}$ & DK & DK & Y & Y & DK & DK & $\mathrm{N}$ & Y & DK & Y & Y & 5 \\
\hline Saltzberg et al. ${ }^{39}$ & DK & DK & Y & Y & Y & Y & Y & Y & Y & Y & $\mathrm{N}$ & 8 \\
\hline Sarma et al. ${ }^{40}$ & DK & DK & $\mathrm{N}$ & Y & $\mathrm{N}$ & Y & Y & Y & Y & Y & $\mathrm{N}$ & 6 \\
\hline Seabourne et al. ${ }^{41}$ & Y & $\mathrm{N}$ & $\mathrm{N}$ & $N$ & $\mathrm{~N}$ & Y & Y & DK & DK & Y & $\mathrm{N}$ & 4 \\
\hline Stiller et al. ${ }^{42}$ & Y & DK & Y & Y & Y & Y & Y & DK & Y & Y & $\mathrm{N}$ & 8 \\
\hline Sukhotnik ${ }^{45}$ & $\mathrm{~N}$ & DK & DK & DK & DK & DK & Y & DK & DK & DK & DK & 1 \\
\hline Todd et al. ${ }^{43}$ & $\mathrm{~N}$ & $\mathrm{~N}$ & $\mathrm{~N}$ & Y & Y & Y & Y & DK & Y & Y & $\mathrm{N}$ & 6 \\
\hline
\end{tabular}




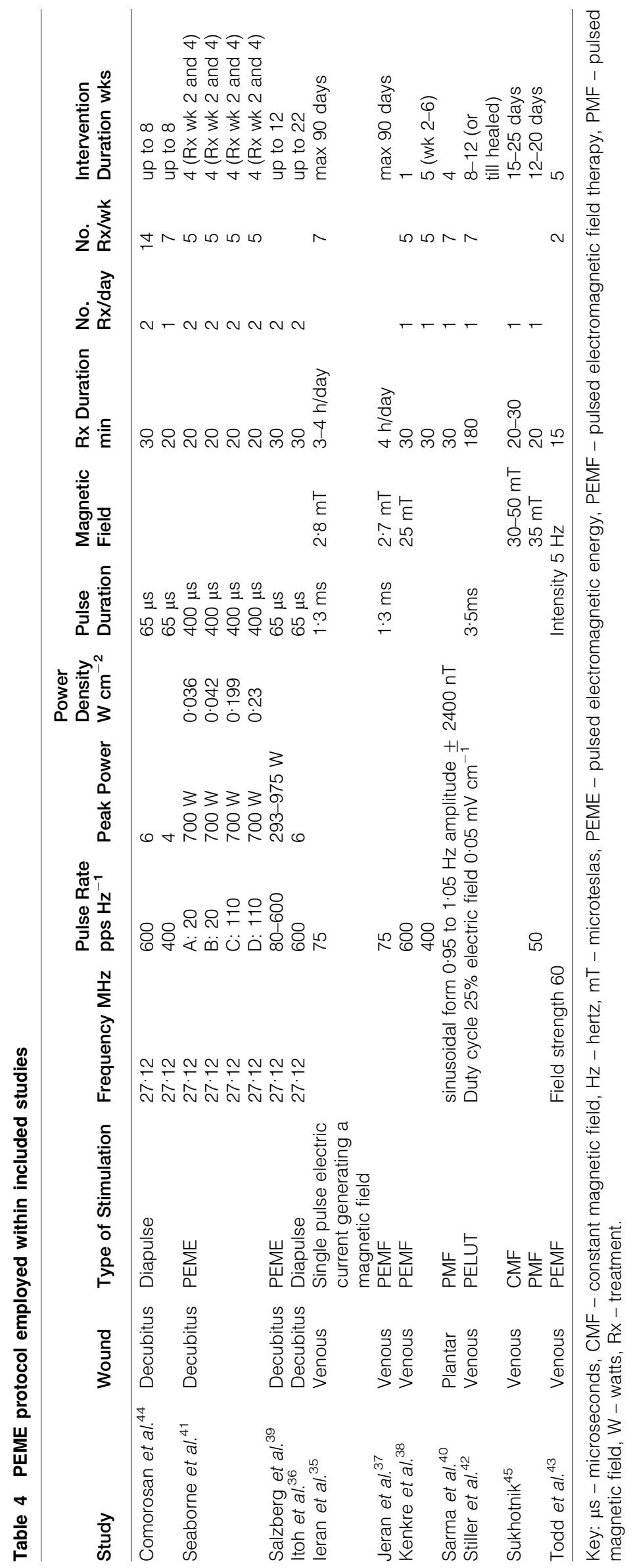




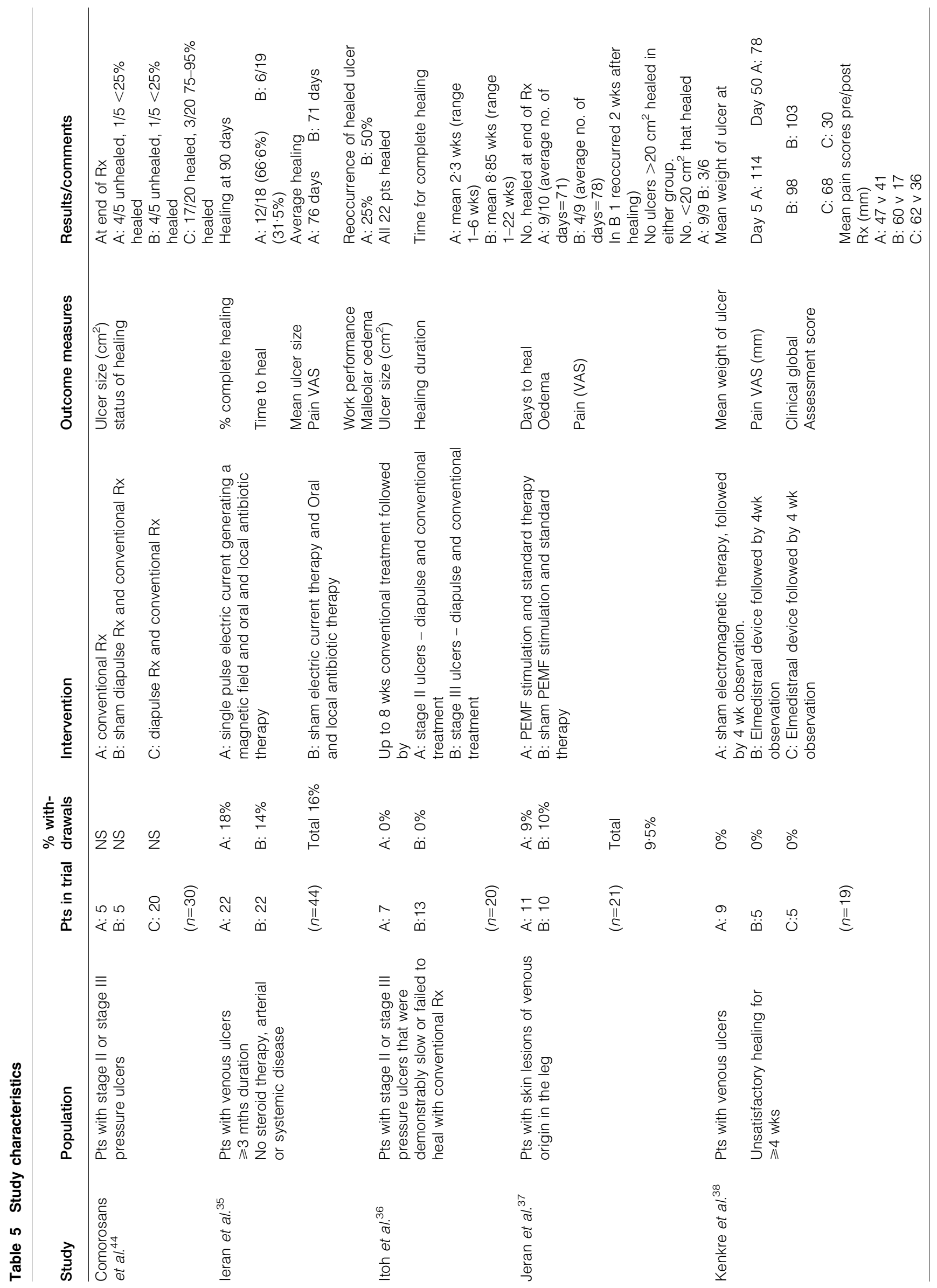




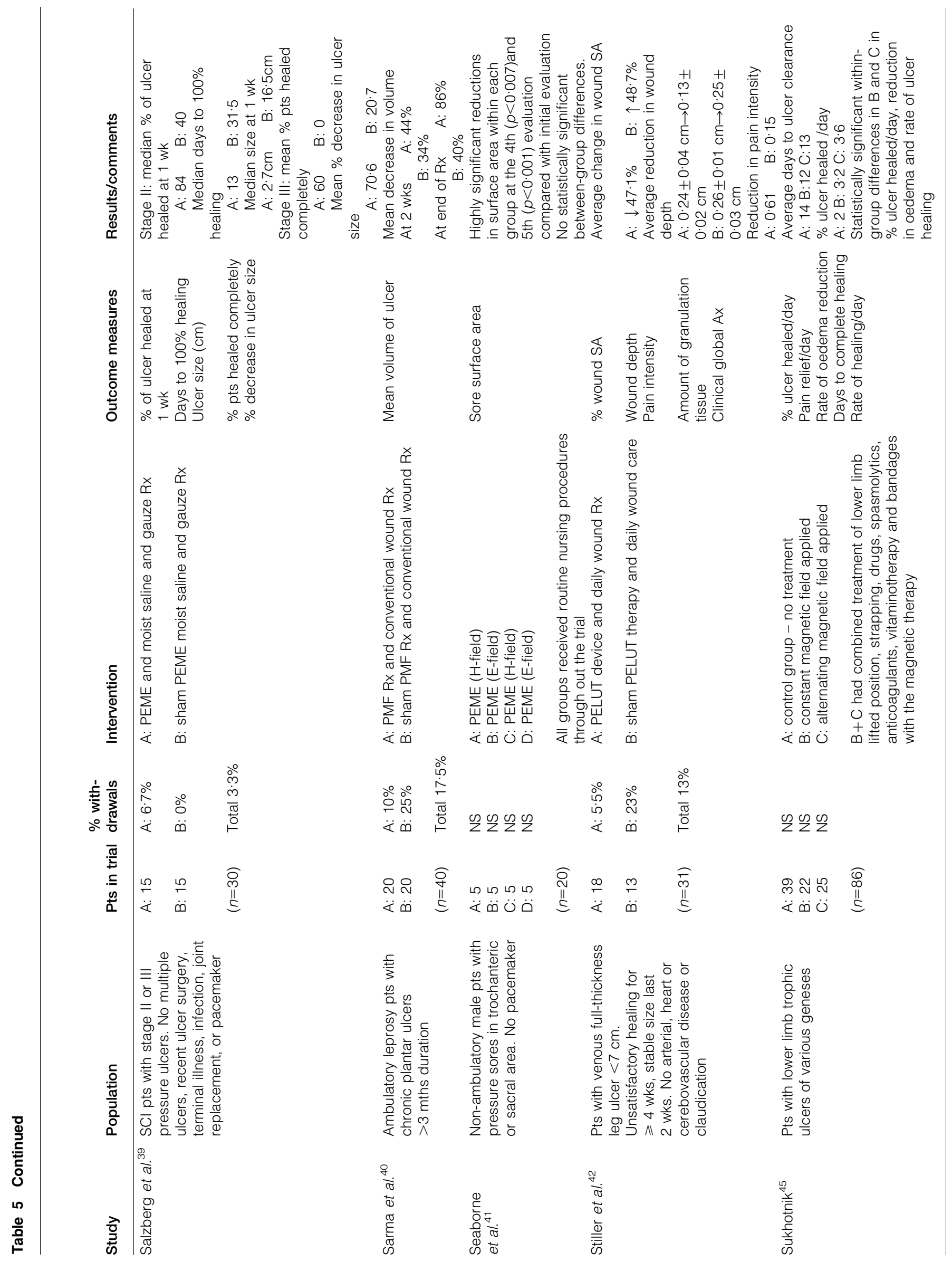




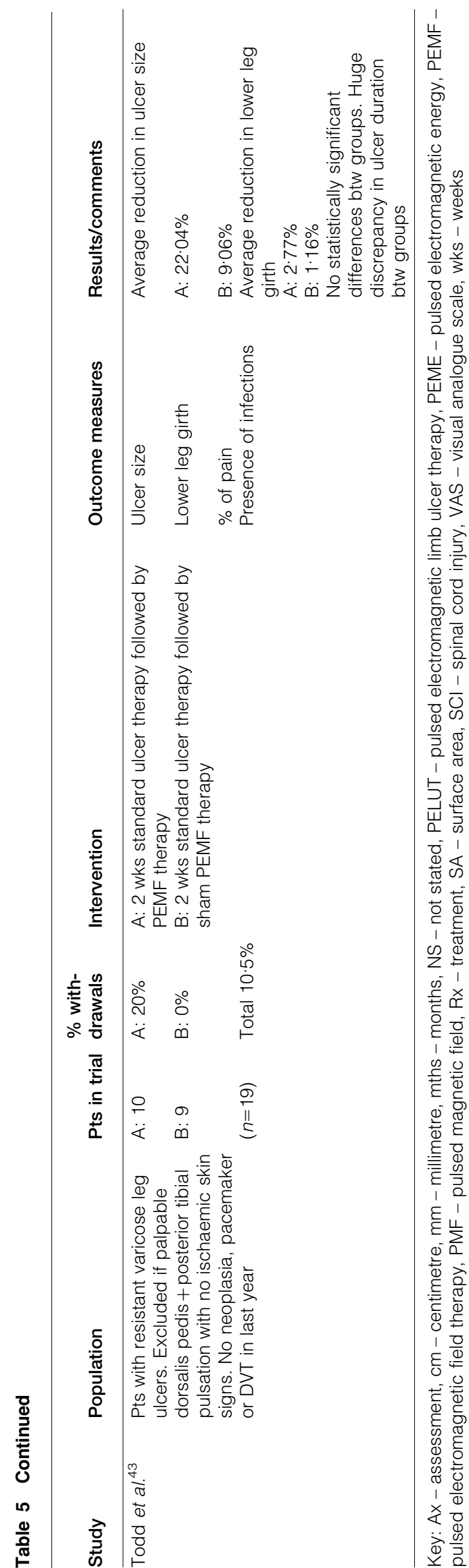

development of cellulitus around ulcer $(n=2)$, diagnosis of rheumatoid arthritis $(n=1)$, patient lost to follow-up $(n=1)$, allergic reaction $(n=1)$ and for one patient no reason was given. Whilst the dropout rates stated were acceptable, it must be noted that the van Tulder scale ${ }^{33}$ only considers overall dropout. Had each group been evaluated independently, the control group short-term attrition rates in two studies ${ }^{40,42}$ would have been deemed unacceptable.

\section{Nature of interventions}

The 11 studies used participants with venous ulcers, ${ }^{35,37,38,42,43,45}$ pressure ulcers ${ }^{36,39,41,44}$ and chronic plantar ulcers. ${ }^{40}$ Table 3 summarises the type, pulse rate, pulse duration, peak power, intensity, and frequency of PEME; the duration of each treatment; number of treatments per day, the number of treatments per week and the total duration of the treatment.

In total five different forms of electromagnetic energy were applied; PEME (diapulse), PEMF therapy, pulsed electromagnetic limb ulcer therapy, pulsed magnetic field (PMF) therapy and a single pulse of electric current that generates a magnetic field. The duration and frequency of interventions were not consistent across the studies. The treatment per day ranged from $15 \mathrm{~min},{ }^{43}$ to $4 \mathrm{~h},{ }^{35,37}$ and number of treatments per week from 2 to 14 sessions. The greatest difference was in the total number of hours in which a form of pulsed electromagnetic therapy was applied. This varied from as little as $2 \cdot 5 \mathrm{~h},{ }^{43}$ to as much as $360 \mathrm{~h}$ of therapy ${ }^{35,37}$ In regard to the treatment protocols there were a few similarities. Seven studies' treatment sessions lasted between 20 and $30 \mathrm{~min}$ each $^{36,38-41,43,44}$ and two studies $^{35,37}$ reported that their sessions can last up to $4 \mathrm{~h}$. However, it was not reported if the $4 \mathrm{~h}$ treatment was continuous or intermittent throughout the day. With the exception of three studies ${ }^{38,41,43}$ treatments were performed every day throughout the trial.

The parameters of the electromagnetic therapy employed also varied. Four studies ${ }^{36,39,41,44}$ used PEME (diapulse) at a frequency of $27 \cdot 12 \mathrm{MHz}$ with the pulse rate ranging from 20 to 600 pulses per second (pps) and the pulse duration from 65 to $400 \mu$ s. While two of the studies ${ }^{36,44}$ used one of the six peak power settings on the machine; one study ${ }^{41}$ used a peak power of $700 \mathrm{~W}$ and the remaining study $^{39}$ did not specify, stating it ranged from 293 to $975 \mathrm{~W}$.

Four studies ${ }^{37,38,43,45}$ evaluated the effect of PEMF therapy and one ${ }^{35}$ used a single pulse electric current generating a magnetic field. Two studies ${ }^{35,37}$ were 
very similar; using the same pulse rate (75 pps); pulse duration $(1.3 \mu \mathrm{s})$ and treatment duration, with the only disparity a magnetic field difference of $0.1 \mathrm{mT}$. The earlier study ${ }^{37}$ appears to be a preliminary report of the study conducted and the latter the complete findings. In comparison, two studies ${ }^{38,45}$ intervention parameters included magnetic fields of 25 and $35 \mathrm{mT}$ respectively and a pulse rate of $600 \mathrm{pps},{ }^{38}$ and frequency of $50 \mathrm{~Hz}^{45}$ The lack of homogeneity present within the studies is highlighted further with three studies ${ }^{40,42,43}$ reporting parameters such as sinusoidal form, amplitude, electric field, duty cycle and field strength. Although all studies implemented a type of pulsed electromagnetic energy, the heterogeneous nature of the programmes implemented makes comparisons difficult. All studies explicitly stated the goal of stimulating healing in chronic ulcers by using electromagnetic therapy.

\section{Effectiveness of treatment}

Pulsed electromagnetic therapy in treating venous ulcers Four high quality studies ${ }^{35,37,42,43}$ (three RCTs, one controlled trial) and two low quality studies ${ }^{38,45}$ (one RCT, one controlled trial) evaluated the effect of pulsed electromagnetic therapy on venous ulcers. Five studies ${ }^{35,37,38,42,43}$ compared PEME with sham PEME, reporting better outcomes (i.e. number of ulcers healed; the reduction in ulcer size and time to complete healing) for the active group. The remaining study $^{45}$ compared the efficacy of constant and alternating magnetic fields in the treatment of trophic ulcers, although this study is of very low quality. One study $^{37}$ failed to perform any statistical analysis and another failed to perform between-group analysis. ${ }^{45}$ Of those that performed an analysis only one found no statistically significant $(p<0 \cdot 05)$ between-group differences following treatment. ${ }^{43} \mathrm{~A}$ number of studies reported statistically significant betweengroup $(p<0.05)$ improvements in the success rate of healing at 90 days, the overall healing at 1 year, ${ }^{35}$ wound surface area at 8 weeks, global assessment score, wound depth, ${ }^{42}$ pain intensity ${ }^{38,42}$ and overall healing ${ }^{38}$ in favour of the active group. One study ${ }^{38}$ found greater pain relief in the two groups receiving PEMF therapy compared to the control group, but the trend for overall healing was less positive. At the end of treatment (day 30), the placebo group had greater overall healing than a PEMF group $(600 \mathrm{~Hz})$. This was explained by one patient in the active group having a large ulcer with poorly defined boundaries, in what was a small sample size. By day 50, however, both active groups had significantly better mean reduction rates than the placebo. Whilst they all reported positive results, the total treatment time varied from 15 to $360 \mathrm{~h}$. Four studies reported on the

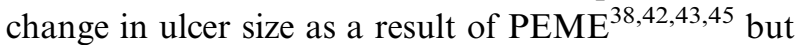
two ${ }^{35,37}$ reported on its ability to completely heal ulcers in a specific time. In the three studies ${ }^{35,37,42}$ where treatment lasted between 3 and $4 \mathrm{~h} / \mathrm{day}$, the participants were taught to apply their own treatment. Participants in the remaining two studies ${ }^{38,43}$ attended a clinical unit.

There was a significant difference in the initial surface area of ulcers, not only between the different studies but also between the groups. In general the greatest improvements in the rate of healing and ulcer size were found in small ulcers (i.e. $<15 \mathrm{~cm}^{2}$ ). One study $^{37}$ found that $4 \mathrm{~h}$ of PEMF therapy for a maximum of 90 days was insufficient to heal any ulcer with a surface area $>20 \mathrm{~cm}^{2}$. In comparison, a study ${ }^{35}$ with similar parameters found that three out of four ulcers $>15 \mathrm{~cm}^{2}$ healed within 9 months following the end of therapy.

PEME was found to be a safe and effective adjunctive therapy, ${ }^{35,42}$ that can provide significant gains in the healing of venous leg ulcers. ${ }^{38}$ Studies concluded that whilst it has demonstrated a positive effect towards treating ulcers ${ }^{37}$ more research is required. ${ }^{37,43}$ There is strong evidence (level 1) that pulsed electromagnetic therapy has a positive effect on wound healing and that it may be more effective than no pulsed electromagnetic therapy in treating ulcers of venous origin.

Pulsed electromagnetic therapy in treating pressure ulcers One high quality $\mathrm{RCT}^{39}$ and three low quality studies $^{36,41,44}$ evaluated the effect of pulsed electromagnetic therapy on pressure ulcers. It was compared with sham PEME treatment in two studies ${ }^{39,44}$ and varying parameters of PEME in another study. ${ }^{41}$ The remaining study used the same parameters of PEME on separate groups of stage II and stage III pressure ulcers, following an 8 week observation period. ${ }^{36}$ All studies continued to use some form of conventional treatment throughout the studies, although this also varied. The 100 participants in these studies had a variety of primary diagnoses: cerebrovascular accident, spinal cord injury, Alzheimer's, organic brain syndrome, spinal cord tumour, diabetes mellitus, spinal stenosis, systemic altherosclerosis and multiple sclerosis.

All patients were treated in a clinical environment for between 20 and $30 \mathrm{~min}$ per session. Two studies $^{36,44}$ did not perform any form of statistical analysis. The only high quality study ${ }^{39}$ found statistically significant between-group differences 
$(p<0 \cdot 05)$ regarding the percentage of ulcer healed at 1 week, the median size of the ulcer at 1 week and the median days to complete healing. ${ }^{39}$ It concluded that PEME therapy combined with standard wound care was significantly more effective at healing ulcers compared to standard treatment alone. One study ${ }^{39}$ found that the rate of healing of grade II pressure ulcers was more than doubled in the active group. It found statistically significant improvements in the percentage healed at 1 week and the median days to complete healing, in the group of stage II pressure ulcers receiving active treatment. In comparison, although grade III ulcers reportedly healed faster in the active group, no statistical analysis was performed for the stage III ulcer group. It must also be noted that the primary outcome measure, the time to $100 \%$ healing, was only assessed in the stage II group. Another study ${ }^{36}$ reported the complete healing of all stage II ulcers (in 1 to 6 weeks) and stage III ulcers (in 1 to 22 weeks) with diapulse treatment, although no statistically significant findings were found. This was following up to 8 weeks of conventional treatment, where no evidence of healing was demonstrated. Also a study ${ }^{41}$ found significant reductions in sore surface area, within four groups receiving various parameters of PEME therapy, but no significant between-group differences at the end of treatment. Despite this it concluded that the positive results found were most likely not due to a placebo effect, time effect, or nursing procedures, but due to the PEME treatment.

Three studies ${ }^{36,41,44}$ used similar program parameters, with the main difference being the duration of the intervention employed. A 22 week study ${ }^{36}$ had a $100 \%$ healing success but in comparison an 8 week study ${ }^{44}$ had $85 \%$ of ulcers healed completely and $15 \%$ of ulcers between 75 and $95 \%$ healed. The main difference was an additional daily dose of PEME at a lower intensity. Studies concluded that PEME is a safe, simple treatment that accelerates wound healing. The low costs of operation ${ }^{44}$ and improved rate of healing can provide significant cost savings and improved patient care, for what in health care is a very expensive problem. ${ }^{36,39,44}$ There is moderate evidence (level 2) that pulsed electromagnetic therapy, combined with conventional wound care will improve the healing of pressure ulcers and that it is more beneficial than conventional wound care alone.

Pulsed electromagnetic therapy in treating plantar ulcers A single high quality $\mathrm{RCT}^{40}$ evaluated the effect of PMF therapy and standard wound care compared with sham PMF therapy and standard wound care, on the rate of healing of plantar ulcers in leprosy patients. Statistically significant within-group differences were found between the volume of ulcer on admission and at the end of treatment for both groups and between admission and the end of week 1 and 2 for the active group. The only significant between-group difference was between admission and the end of treatment, with the ulcer size decreasing by $40 \%$ or more in $53 \%$ of the control compared to $89 \%$ in the PMF group $(p=0.02)$. A decrease of $80 \%$ or more was observed in $33 \%$ of the PMF group and none in the control group $(p=0 \cdot 01)$. The study concluded that exposure to PMF causes a substantially more rapid rate of healing of the ulcers than in those who received conventional wound treatment only. As only one patient managed complete healing at the end of the treatment period (4 to 5 weeks), a larger trial is needed to determine if more patients would have healed with a longer trial. Overall, there is moderate evidence for the use of PMF therapy in the treatment of plantar ulcers in leprosy patients.

\section{Outcome measures}

The mean ulcer surface area was the most common outcome measure used, but the methods and scales utilised varied. Millimeters and centimeters squared were the most common values used, but one study ${ }^{38}$ calculated the area using the weight of sterile acetate sheets that cover the site. There were a variety of outcome measures, with the visual analogue scale measuring pain, the percentage of healing and the days to complete healing the most frequently used. Despite the impact that chronic ulcers can have on patients only two studies ${ }^{35,38}$ assessed the impact that the ulcer had on quality of life/daily activities. Generally, the primary outcome measures used were similar and appropriate for assessing the studies' stated aims.

There are some similarities between trials that evaluate the same form of PEME, but problems arise when comparing one form of therapy to another. Overall 10 studies concluded positive findings, although few statistically significant between-group results were found. Six were of high methodological quality; therefore there is strong evidence to support that PEME has a positive effect in the treatment of chronic wounds, but the extent of this is still unknown.

\section{Discussion}

As previously stated, the overall aim of this study was to evaluate if PEME therapy can accelerate wound healing. Previous reviews have found little supporting evidence on the effect of electromagnetic therapy on 
treating pressure ulcers ${ }^{46}$ venous leg ulcers ${ }^{47}$ or as adjunctive treatments for chronic skin wounds. ${ }^{48}$ Whilst electromagnetic therapy may promote wound healing, one review found that the methodological flaws in how studies were conducted, led to considerable uncertainty as to whether it provides clinically significant health benefits. This is the first review to systematically assess PEME therapy in the treatment of all chronic wounds but the lack of RCTs identified highlights a gap in the literature regarding the use of PEME therapy.

Briefly, 11 studies $(n=360)$ out of 40 were included of which six were of high methodological quality. Methodological scores ranged from one to nine (maximum 11) with a mean score of $5 \cdot 5(\mathrm{SD}=1 \cdot 73)$. For venous ulcers, there is strong evidence that PEME is more effective that sham PEME. For pressure and plantar ulcers, there is moderate evidence that PEME, in combination with conventional wound care is better than conventional wound care alone. The heterogeneity in duration, frequency, voltage and magnetic field made it difficult to make detailed comparisons or specific recommendations regarding its application.

This review is somewhat restricted. The selection of all relevant studies is crucial to the validity of a systematic review ${ }^{33}$ to limit the degree of bias. Due to limited resources the English translation of one study was unable to be obtained therefore excluding a potentially relevant trial. Failure to identify all potentially relevant trials can lead to bias. ${ }^{49}$ In terms of methodological assessment, no attempts were made to contact authors to clarify any absent information, a factor that should be addressed in subsequent reviews. Also, the reviewers were at no point blinded to the authors or journals, which may have led to study bias. A meta-analysis was not undertaken primarily due to the heterogeneous nature of the studies' interventions, and due to the variety of outcome measures used. Therefore this review is limited in its recommendations for clinical practice. Finally, this review did not assess clinical appropriateness or cost-effectiveness, but instead concentrated on statistical significance. It is conceded that statistical significance is not always reflective of clinical significance; this may be a subject for further investigation.

A failure to report on $27 \cdot 3 \%$ of the internal validity criteria clearly highlights an important methodological shortcoming within the studies, thus impeding an accurate estimation of quality. ${ }^{50}$ Despite a mean methodological score of 5.5 the contrast in treatment protocols made comparisons between studies difficult. It should be noted that more journals are accepting the CONSORT statement on enhancing the quality of reporting of RCTs,${ }^{51}$ which will hopefully lead to more methodologically rigorous RCTs, ${ }^{52}$ with greater standardisation. The areas most in need of improvement are description of randomisation, concealed treatment allocation, baseline comparability between the groups, and intention-to-treat analysis. With both the earliest and most recent study being of high methodological quality there wasn't a clear trend relating to study quality and publication date. However, two out of the three most recent studies were of low quality, demonstrating deterioration in methodological quality. As expected the RCTs were superior in quality overall, with two exceptions. ${ }^{38,44}$

To achieve a homogeneous group, participants must be clearly defined in terms of the type and duration of their condition. ${ }^{53}$ The most difficult variable to control in a wound healing trial is that of initial wound size. ${ }^{39}$ In the three studies ${ }^{35,41,42}$ that performed an adequate method of randomisation only one study ${ }^{42}$ demonstrated baseline comparability between the groups. Due to the small number of participants involved and the large degree of variability between study populations, any randomisation process will not provide an even distribution of size. In addition, the small sample sizes can give rise to type II error by reducing the power of statistical analysis. ${ }^{54}$ This error can be decreased by increasing the sample size; ${ }^{54}$ however, it may be difficult to obtain a large number of participants with similar sized ulcers.

Poor baseline comparability can severely affect the results of any trial. This was shown in one trial, ${ }^{43}$ where the large initial ulcer size of just one patient resulted in huge baseline differences between the groups. With varying results on the treatment of large ulcers more research is required. The duration of the ulcer is another important variable affecting trials, but the question of whether PEME therapy provides greater benefit to ulcers of shorter or more prolonged duration is left unanswered. Studies including ulcers of both short and prolonged durations yielded positive results. One study ${ }^{38}$ had participants with ulcer durations ranging from seven months to a massive 49 years, but from their results it was not possible to ascertain which patients benefited most.

Within clinical practice PEME therapy is commonly combined with conventional, routine or ancillary wound care. The specific type of standard care varies between studies, making it difficult to 
determine the value of PEME alone. Many participants received standard wound care prior to the trial, continuing it throughout, although usually at a different frequency/type to what they had previously received. In a study ${ }^{36}$ where participants were used as their own controls, it is possible that the conventional treatment, when it interacted with PEME therapy, jump-started the healing process. It must be taken into account that the improved healing time may be due to the previous treatment or the fact that participants in trials often show better results and receive better medical care. Also when studies ${ }^{38,44}$ use various parameters of PEME therapy on each patient, it is impossible to differentiate between them. The effect that various levels of PEME have on chronic wounds of different sizes must be considered in future studies to enable the optimal treatment to be found.

The nature of PEME therapy means that there are a number of safety implications to address. These include hazards such as burns or cardiac failure and contraindications such as metal in the tissues or impaired thermal sensation. ${ }^{2}$ A previous study on osteoarthritic hip and knee pain felt that too low a dose of PEME will have no effect and too high a dose may be detrimental. ${ }^{55}$ Despite the different dosages implemented in this review the majority of studies reported no adverse effects. The only reported incidents were of severe headaches, heat sensation, tingling and pins and needles in the lower $\operatorname{limb}^{38}$ and the development of cellulitus. ${ }^{43}$

The most clinically important outcome in evaluating treatment for wound healing is the percentage of participants that heal completely, ${ }^{48}$ an outcome that only three of the studies ${ }^{35,39,44}$ managed to assess. It is imperative that groups undergo the same assessments and statistical analysis throughout the study. If possible a long-term follow-up should be performed to fully assess the effectiveness of the intervention and to discover the number of ulcers that reoccur. There is need for greater standardisation in the outcome measures and scales that studies used to assess patients to enable more comparisons to be made across studies. In addition, the length of treatment is a controversial issue that needs investigating further. Positive results were found in both short and long trials, therefore the parameters of therapy implemented need to be studied in detail. Only by altering and studying one variable at a time, can the full potential of PEME therapy in wound healing be discovered. When deciding on the duration of a study, the cost of the therapy must also be considered.
Standard wound care places a considerable financial burden on patients, their families and the healthcare system. At an annual estimated cost of between $1 \cdot 4$ and $2 \cdot 1$ billion pounds a year to treat pressure ulcers ${ }^{4}$ and approximately 400 million pounds a year to treat venous leg ulcers, ${ }^{6}$ the financial implications that an improved rate of healing offers are significant. One study states that the cost of changing a wound dressing three times a day for a week is more than double the cost of applying two 30 min PEME treatments every day for a week. ${ }^{36} \mathrm{~A}$ review on the cost of treating foot ulcers found that the greatest expenses were for staff and transportation, and it recommends reducing costs by reducing the frequency of dressing changes. ${ }^{56}$ Two studies ${ }^{35,42}$ in this review used portable units to apply the electromagnetic energy at home. Following a demonstration and clear instruction the patients applied their own treatment each day. Both studies reported significant improvements in the rate of healing following PEME treatment; therefore, not only does this reduce the number of dressing changes required but the self-administration of the treatment saves valuable money on staff and transportation. The nature of the condition means that many patients are permanently based in a clinical facility and may not be fit enough to treat themselves. However, the potential benefit that a portable device offers cannot be underestimated.

\section{Recommendations}

Further research is required to help establish appropriate treatment parameters for practice. Future RCTs should be more vigilant in their methodological quality, particularly concerning the method of randomisation used, including an intention-to-treat analysis and concealment of treatment allocation. Issues of baseline comparability, blinding the care provider and sample sizes also need to be addressed. Studies should use larger sample sizes to determine the level of effectiveness. ${ }^{39,41}$ Furthermore, all methods should be thoroughly reported to allow more accurate evaluation. Trials should include outcome measures that promote homogeneity and comparability between trials. Assessments should be carried out over prolonged periods to determine long-term and therefore more meaningful effects on this chronic condition. It is imperative that trials try to assess ulcers of similar origin, duration and size. Different parameters of pulsed electromagnetic therapy must be investigated, each with their own control to determine which is more effective. To achieve these 
aims a greater number of high quality RCTs need to be undertaken.

In conclusion, there is strong evidence to suggest that PEME has a positive effect on the rate of wound healing but the degree to which it is clinically significant is less conclusive. It is felt that a detailed and unbiased evaluation of the literature was undertaken. Despite a limited number of statistically significant between-group differences, all of the studies agree that the healing rate was accelerated. However, due to the heterogeneous nature of the interventions and numerous methodological flaws the authors could make no definitive recommendations for clinical practice.

Further research is required to addresses these inadequacies and adhere to the recommendations made. Only then can the true value of PEME be determined, which may in the future allow for a metaanalysis and therefore more meaningful conclusions for clinical practice.

\section{References}

1 Fernandez-Chimeno M, Houghton P, Holey L. Electrical stimulation foe chronic wounds (Cochrane Review). In: The Cochrane Library, Issue 1, 2004. Oxford: Update Software

2 Kitchen S. Electrotherapy: Evidence-Based Practice, 11th edn. New York: Churchill-Livingstone, 2002

$3^{*}$ European Pressure Ulcer Advisory Panel. Guideline on treatment of pressure ulcers. EPUAP Review 1999;1:31-3

4 Bennett G, Dealey C, Posnett J. The cost of pressure ulcers in the UK. Age and Aging 2004;33:230-5.

5 National Health Service Centre for Reviews and Dissemination. Compression therapy for venous leg ulcers. Effective Health Care Bulletin 1997;3:1-12

6 Simon DA, Dix FP, McCollum CN. Management of venous leg ulcers. British Medical Journal 2004;328:1358-62

7 Charles H. The impact of leg ulcers on a patients' quality of life. Professional Nurse 1995;10:571-2

8 Bosanquet N. Costs of venous leg ulcers - from maintenance therapy to investment programs. Phelobology 1992;7:44-6

9 Nelson EA, Bradley MD. Dressings and topical agents for arterial leg ulcers (Cochrane Review). In: The Cochrane Library, Issue 3, 2003. Oxford: Update Software

10 Mekkes JR, Loots MAM, van Der Wal AC, Bos JD. Causes, investigation and treatment of leg ulceration. British Journal of Dermatology 2003;148:388-401

11 Belcaro G, Sager P, Borgwardt A, Holm A, Jelnes R, Rosenkvist L, Possati F. Arterial pressure measurements correlated to symptoms and signs of peripheral arterial disease. Acta Chirurgica Belgica 1983;83:320-6

12 Harding KL, Morris HL, Patel GK. Healing chronic wounds. British Medical Journal 2002;324:160-3

13 Waldrop J, Doughty D. Wound healing physiology. Acute and Chronic Wounds Nursing Management. St Louis: Mosby Inc, 2000

14 Stadelman WK, Digenis AG, Tobin GR. Impediments to wound healing. American Journal of Surgery 1998;176:39S-47S

$15^{*}$ Vodovnik L, Karba R. Treatment of chronic wounds by means of electric and electromagnetic fields. Biological Engineering and Computing 1992;30:257-66

$16 \overline{\text { Burr HS, Taffel }} \overline{\mathrm{M}}$, Harvey SC. An electronic study of the healing wound in man. Journal of Biological Medicine 1940;12:483-5

17 Clayton EB. Clayton's Electrotherapy, 10th edn. London: Saunders, 1996

18* Hayne CR. Pulsed high frequency energy - its place in physiotherapy. Physiotherapy 1984;70:459-466
19 Kitchen S, Partridge C. Review of shortwave diathermy continuous and pulsed patterns. Physiotherapy 1992;78:243-52

20* Oliver DE. Pulsed electro-magnetic energy - what is it? Physiotherapy 1984;70:458

21 Low J, Reed A. Electrotherapy Explained: Principles and Practice, 2nd edn. Oxford: Butterworth-Heinemann, 1994

22 Low J. Dosage of some pulsed shortwave clinical trials. Physiotherapy 1995;81:611-6

23 Goldin JH, Broadbent NR, Nancarrow JD, Marshall T. The effects of Diapulse on the healing of wounds: a double-blind randomised controlled trial in man. British Journal of Plastic Surgery 1981;34:267-70

24 Wyper DJ, McNiven DR. Effects of some physiotherapeutic agents on skeletal muscle blood flow. Physiotherapy 1976;63:83-8

25 Robertson VJ, Spurritt D. Electrophysical agents: implications of their availability and use in undergraduate clinical placements. Physiotherapy 1998;84:335-44

26 Pope GD, Mockett SP, Wright JP. A survey of electrotherapeutic modalities: ownership and use in the NHS in England. Physiotherapy 1995;81:82-91

27 Higgins JPT, Green S. Cochrane Handbook for Systematic Reviews of Interventions version 4.2.5 (Cochrane Review). In: The Cochrane Library, Issue 3, 2005. Oxford: Update Software

28* van Tulder MW, Furlan A, Bombardier C, Bouter LM, the editorial board of the Cochrane Collaboration Back Review Group. Updated method guidelines for systematic reviews in the Cochrane Collaboration Back Review Group. Spine 2003;28:12908

29* van Tulder MW, Assendelft WJ, Koes BW, Bouter LM, the editorial board of the Cochrane Collaboration Back Review Group. Method guidelines for systematic reviews in the Cochrane Collaboration Back Review Group for Spinal Disorder. Spine 1997;22:2323-30

30 Jadad AR, Moore A, Carroll D, Jenkinson C, Reynolds DJ, Gavaghan DJ, McQuay HJ. Assessing the quality of reports of randomised clinical trials: is blinding necessary? Controlled Clinical Trials 1996;17:1-12

31 Verhagen AP, de Bie RA, Lenssen AF, de Vet HC, Kessels AH, Boers M, van Den Brandt PA. Quality assessment of trials: a comparison of three criteria lists. Physical Therapy Reviews 2000;5:49-58

32 Sherrington C, Herbert RD, Maher CG, Moseley AM. PEDro: a database of randomised controlled trials and systematic reviews in physiotherapy. Manual Therapy 2000;5:223-6

33 van Tulder MW, Malmivaara A, Esmail R, Koes B. Exercise therapy for low back pain: a systematic review within the framework of the Cochrane Collaboration Back Review Group. Spine 2000;25:2784-96

34 Moher D, Cook DJ, Eastwood S, Olkin I, Rennie D, Stroup DF et al. Improving the quality of reports of meta-analyses of randomized controlled trials: the Quorum Statement. The Lancet 1999;354:1896-900

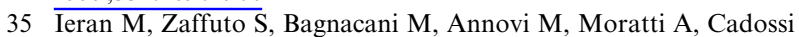
R. Effect of low frequency pulsing electromagnetic fields on skin ulcers of venous origin in humans: a double-blind study. Journal of Orthopaedic Research 1990;8:276-82

36* Itoh M, Montemayor JS, Matsumoto E, Eason A, Lee MH, Folk FS. Accelerated wound healing of pressure ulcers by pulsed high peak power electromagnetic energy (diapulse). Decubitus 1991;4:24-5, 29-34

37* Jeran M, Zaffuto S, Moratti A, Bagnacani M, Cadossi R. PEMF stimulation of skin ulcers of venous origin in humans: preliminary report of a double blind study. Journal of Bioelectricity 1987;6:1818

38 Kenkre JE, Hobbs FDR, Carter YH, Holder RL, Holmes EP. A randomised controlled trial of electromagnetic therapy in the primary care management of venous leg ulceration. Family Practice 1996;13:236-41

39 Salzberg CA, Cooper-Vastola SA, Perez F, Viehbeck MG, Byrne DW. The effects of non-thermal pulsed electromagnetic energy on wound healing of pressure ulcers in spinal cord-injured patients: a randomised, double-blind study. Ostomy Wound Management 1995;41:42-44, 46, 48

40 Sarma GR, Subrahmanyam S, Deenabandhu A, Babu CR, Madhivathanan S, Kesavaraj N. Exposure to pulsed magnetic 
fields in the treatment of plantar ulcers in leprosy - a pilot randomised, double-blind, controlled clinical trial. Indian Journal of Leprosy 1997;69:241-250

41 Seaborne D, Quirion-DeGirardi C, Rousseau M, Rivest M, Lambert J. The treatment of pressure sores using pulsed electromagnetic energy (PEME). Physiotherapy Canada 1996;48:131-7

42 Stiller MJ, Pak GH, Shupack JL, Shaler S, Kenny C, Jondreau L. A portable pulsed electromagnetic field (PEMF) device to enhance healing of recalcitrant venous ulcers: a double-blind, placebocontrolled clinical trial. British Journal of Dermatology 1992;127:147-54

43 Todd DJ, Heylings DJA, Allen GE, McMillin WP. Treatment of chronic varicose ulcers with pulsed electromagnetic fields: a controlled pilot study. Irish Medical Journal 1991;84:54-5

44 Comorosan S, Vasilco R, Arghiropol M, Paslaru L, Jieanu V, Stelea S. The effect of diapulse therapy on the healing of decubitus ulcer. Romanian Journal of Physiology 1993;30:41-5

45 Sukhotnik IG. Comparative effectiveness of using constant and alternating magnetic fields in the treatment of trophic ulcers. Vestnik Khirurgii Imeni-i-i-Grekova 1990;144:123-4

46 Olyaee Manesh A, Flemming K, Cullum NA, Ravaghi H Electromagnetic therapy for treating pressure ulcers (Cochrane Review). In: The Cochrane Library, Issue 2, 2006. Oxford: Update Software

47* Ravaghi H, Flemming K, Cullum N, Olyaee Manesh A Electromagnetic therapy for treating venous leg ulcers (Cochrane Review). In: The Cochrane Library, Issue 2, 2006. Oxford: Update Software
48 Electrical Stimulation or Electromagnetic Therapy as Adjunctive Treatments for Chronic Skin Wounds. Chicago, IL: Blue Cross and Blue Shield Association, 2005;20

49 Egger M, Ebrahim S, Smith GD. Where now for meta-analysis? International Journal of Epidemiology 2002;31:1-5

50 de Vet HCW, de Bie RA, van der Heijden GJMG, Verhagen AP, Sijpkes P, Knipschild PG. Systematic reviews on the basis of methodological criteria. Physiotherapy 1997;83:284-9

51 Begg C, Cho M, Eastwood S, Horton R, Moher D, Olkin I, Pitkin R, Rennie D, Schulz KF, Simel D, Stroup DF. Improving the quality of reporting of randomized controlled trials: the CONSORT statement. JAMA 1996;276:637-9

52 de Vet HCW, de Bie RA, van der Heijden GJMG, Verhagen AP, Sijpkes P, Knipschild PG. Systematic reviews on the basis of methodological criteria. Physiotherapy 1997;83:284-9

53 Harte AA, Baxter GD, Gracey JH. The efficacy of traction for back pain: a systematic review of randomised controlled trails. Archives of Physical Medicine and Rehabilitation 2003;84:154253

54 Polgar S, Thomas SA. Introduction to Research in the Healt Sciences, 4th edn. Sydney: Churchill Livingstone, 2000;63, 278

55 Klaber Moffett JA, Richardson PH, Frost H, Osborn A. A placebo controlled double blind trial to evaluate the effectiveness of pulsed short wave therapy for osteoarthritic hip and knee pain. Pain 1996;67:121-7

56 Apelqvist J, Ragnarson-Tennvall G, Larisson J. Topical treatment of diabetic foot ulcers: an economic alanysis of treatment alternatives and strategies. Diabetic Medicine 1995;12:123-8 